\title{
A Retrospective Study of Dogs with Osteomyelitis Secondary to Fractures (2016-2020)
}

\author{
Cornel IGNA ${ }^{1 *}$, Larisa SCHUSZLER ${ }^{1}$, Daniel BUMB ${ }^{1}$, Roxana DASCALU ${ }^{1}$, Cristian ZAHA $^{1}$, Bogdan SICOE \\ ${ }^{1}$ Banat's University of Agricultural Science and Veterinary Medicine, "King Mihai I of Romania" from \\ Timisoara, 119 Calea Aradului, Timisoara 300645, Romania \\ * corresponding author: ignacornel@gmail.com
}

Bulletin UASVM Veterinary Medicine 77(2)/2020

Print ISSN 1843-5270; Electronic ISSN 1843-5378

doi:10.15835/buasvmcn-vm:2020.0013

\begin{abstract}
The purpose of this work was to evaluate the role of surgical procedures and the effectiveness of antimicrobial therapy in dogs with osteomyelitis secondary to fractures repair.

Medical records between 2016 and 2020 were analyzed. Clinical data including signalment, affected bone segment, fracture type, isolated microorganisms, antimicrobial susceptibility profiles, applied therapeutic management and fracture healing time were documented.

Eleven cases were confirmed with exogenous osteomyelitis of long bones secondary to fractures repair. The most common microorganisms isolated were Staphylococcus spp., Streptoccocus spp., and Escherichia coli. Amoxicillin and clavulanate potassium followed by ceftriaxone were the most effective drugs. High-resistance rates were documented for penicillin and clindamycin. All 10 cases undergoing surgical revision were cured in 10-17 weeks.

Simultaneous debridement with implant removal, reduction, and rigid internal fracture fixation is a satisfactory method for revision surgery in treatment of long bones fractures complicated with osteomyelitis.
\end{abstract}

Keywords: dog, fracture, osteomyelitis, treatment

\section{Introduction}

The term osteomyelitis, which literally means inflammation of bone, including the marrow and cortex (Anderson, 2016), is most commonly applied to bacterial or fungal infections of bone (Budesberg, 2012; Lio et al., 2012; May, 2002; Quinn et al.; 2011, Schulz, 2007).

Acute osteomyelitis is rare and generally does not show detectable radiographic changes until 5 to 10 days after bone inoculation. Chronic osteomyelitis is seen as a complication from orthopedic surgery. Osteomyelitis can mimic other diseases such as panosteitis, hypertrophic osteodystrophy, and neoplasia and should be differentiated from these (Callum, 2006). Osteomyelitis associated with fracture has been described in dogs (Johnson et al., 1984; Woodard and Riser, 1991). Posttraumatic osteomyelitis is one of the most serious complications after fracture treatment (Soontonvipart et al., 2003).

In general, osteomyelitis develops focally as a sequela to penetrating trauma or as a surgical complication (Rabillard et al., 2011). Osteomyelitis may be either exogenous or hematogenous in origin (Budesberg, 2012; Emmerson and Pead, 1999; Jackson and Pacchiana, 2004; Siqueira et al., 2014; Soontornvipart et al., 2003). Usually the exogenous route occurs during open reduction of a closed fracture or is associated with an open fracture (Braden, 1991; Jackson and Pacchiana, 2004). In these cases, the bacterial contamination is the most prevalent cause of osteomyelitis and 
more commonly associated with Staphylococcus species (Budesberg, 2012; Caywood, 1983; Piermattei et al.; 2006, Simionato et al., 2003; Siqueira et al., 2014; Soontornvipart et al., 2003).

Imaging methods (radiography, computed tomography, fistulography, and ultrasonography) have been used to detect signs of bone damage caused by osteomyelitis (Braden, 1991, Buttin et al., 2013, Caywood, 1983, Fossum and Hulse, 1992, Siqueira et al., 2014). Dvorak et al. (2000) shows that about $30 \%$ of $40 \%$ of the patients who had complications after osteosynthesis had abnormal radiological bone healing without any abnormal clinical signs.

The definitive diagnosis and the treatment of post-traumatic osteomyelitis is based on the microbiological cultures and in vitro antimicrobial susceptibility testing (Caywood, 1983; Braden, 1991; Jackson and Pacchiana, 2004; Bubenik, 2005; Schulz, 2007; Simionato et al., 2003; Siqueira et al., 2014). Specimens for culture should be obtained by needle aspiration or bone biopsy rather than tract drainage (Fossum and Hulse, 1992; Jackson and Pacchiana, 2004; Piermattei et al., 2006; Schulz, 2007).

Shirtliff et al. (2002), Soontonvipart et al. (2003) and Stein et al. (1998) find that at the patients at high risk of osteomyelitis development or in the patients where the infection is already present, it is important to know hospital-specific pathogens to select adequate complementary antibiotics. This emphasizes the need of epidemiologic studies, specific for each clinic.

The aim of this study was the retrospective evaluation of the role of surgical procedures, foreign body reaction and the effectiveness of local antimicrobial therapy in dogs with osteomyelitis secondary to fractures registered in the Surgery Clinic of the Faculty of Veterinary Medicine fromTimisoara during 2016-2020.

\section{Materials and methods}

Were analyzed retrospectively the medical records of the Surgery Clinic of Banat's University of Agricultural Sciences and Veterinary Medicine, Timisoara, Romania between 2016 and may 2020 to identify dogs that had osteomyelitis in long bones.

The analysis was based on the diagnostic records of individual dogs with bone pathology. The criteria for including the dogs in the study were based on preoperative clinical and radiographic examinations, as well as on microbiological cultures and antimicrobial susceptibility testing that confirmed the diagnosis of osteomyelitis secondary to fractures.

The microbiological cultures and antimicrobial susceptibility testing were done in the Laboratory of the Infectious Diseases Clinic of Banat's University of Agricultural Sciences and Veterinary Medicine, Timisoara, Romania. Culture material was obtained from bone biopsy specimens, needle aspiration of the fracture site or bone implants.

Data recorded and analyzed for each patient included: breed, age, body weight, sex, affected bone segment, causes of the injury (fracture), fracture types (open, closed), the isolated microorganisms, antimicrobial susceptibility profiles, systemic and/ or local antibiotic prophylaxis and postoperative therapy, applied therapeutic management (incision, drainage, surgical debridement, type of surgical fracture fixation chosen (open reduction and internal fixation - ORIF with intramedullary pin or plate, or liniar external fixator - FEL), or limb amputation, and fracture healing time.

\section{Results and discussions}

Total number of dogs admitted to our clinic during 2016 - 2020 was 7271 . Out of this number, 329 dogs $(0.45 \%)$ represented cases with fractures treated surgically by osteosynthesis. Injuries were caused in $64 \%$ of the cases by a motor vehicle accident, and in $18 \%$ of cases (four patients) by dog bites. In eleven cases (3.34\%) was confirmed the diagnosis of osteomyelitis secondary to fractures repairs, from which 3 dogs were initially treated in our clinic (27\%) and 8 dogs in another clinic $(73 \%)$. Exogenous osteomyelitis was the result of postoperative infection of open fractures $(7 / 64 \%)$ or closed fractures $(4 / 36 \%)$. Fractures were located in the femur $(2 / 18 \%)$, humerus $(2 / 18 \%)$, tibia $(5 / 46 \%)$, and radius and ulna $(2 / 18 \%)$. Fracture fixation was initially done with intramedullary pins $(3 / 27 \%)$, plates $(4 / 39 \%)$, and FEL $(4 / 39 \%)$.

Radiographs showed signs of multiple areas of trabecular osteolysis of the long bones (osteolytic remodeling), the osteolysis around the screws, and heterogenous radiodensity of the medullary cavity along the bone diaphysis (Figure 1). 
The intraoperative findings showed in all cases nonunion, purulent material or foreign bodies, and fistulas (Figure 2).

Eleven microbiological cultures from canine osteomyelitis were evaluated in the present study. From the total number of samples, $82 \%$ were cultured for aerobes versus $4 \%$ anaerobes, and $4 \%$ were cultured for both anaerobes and aerobes. The most common microorganisms isolated from aerobic cultures were one of the following bacteria: Staphylococcus spp., Streptoccocus spp., and Escherichia coli (82\%), followed by anaerobes cultures with Bacteroides spp. (4\%) and both anaerobes and aerobes cultures with Escherichia coli + beta-hemolytic Staphylococcus + Bacteroides (4\%).

The most effective drugs against isolated bacteria were amoxicillin and clavulanate potassium (79\%) followed by ceftriaxone (71\%). High-resist-

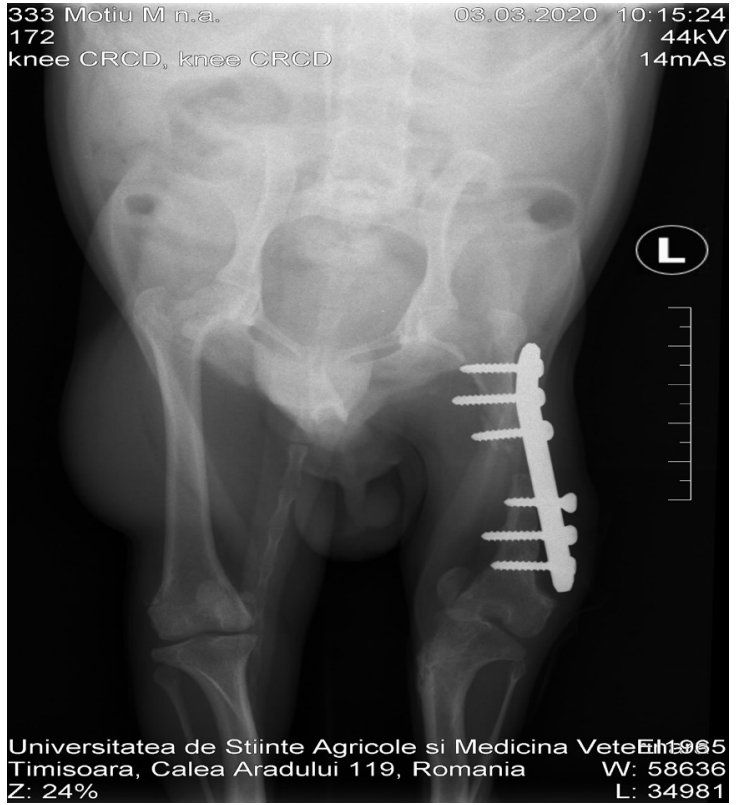

a

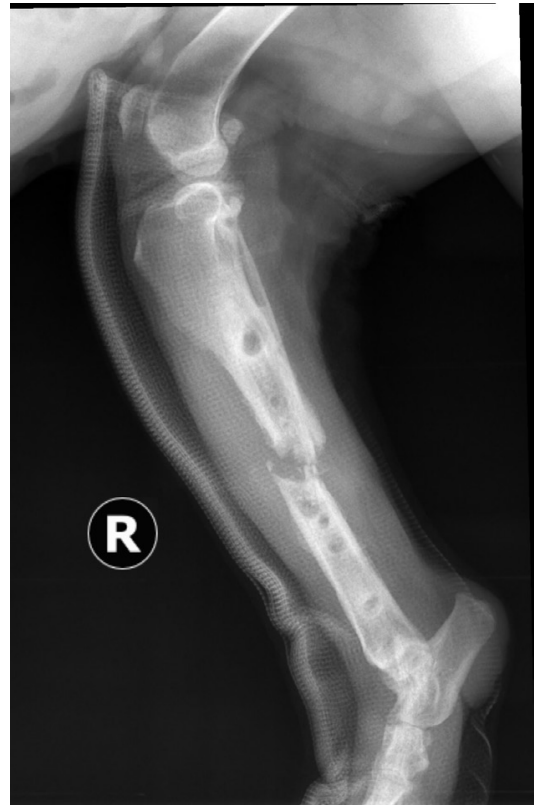

b

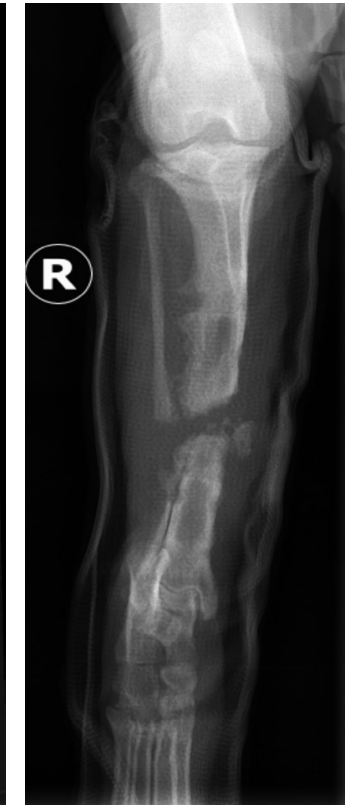

C

Figure 1. Radiograph of osteomyelitis-fracture treated with plate: femur (a), tibia - mediolateral view (b) and ventrodorsal view (c)

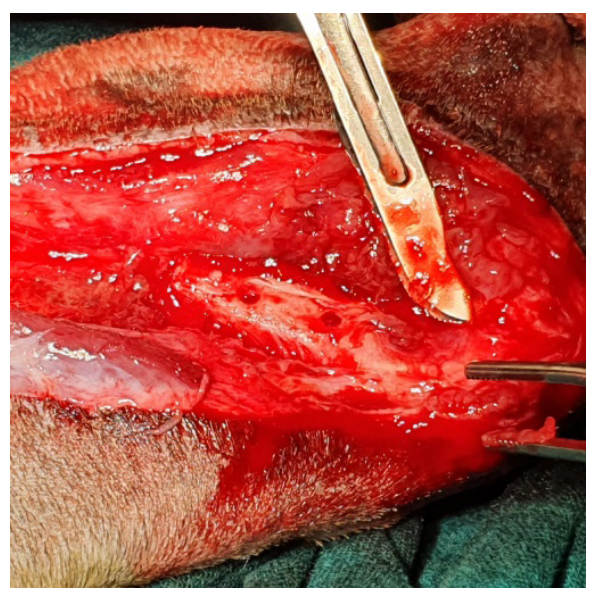

a

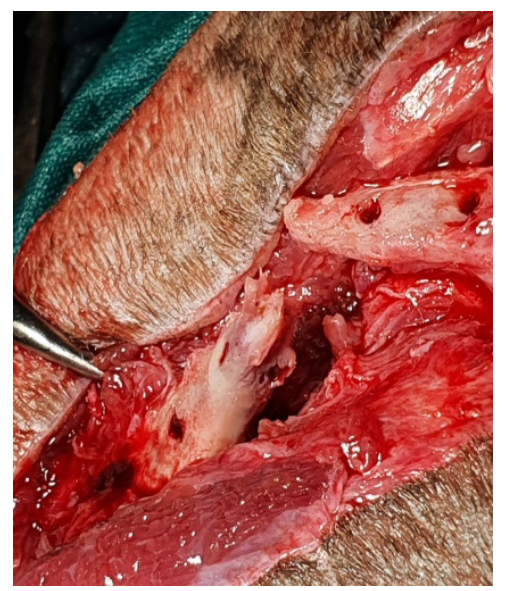

b

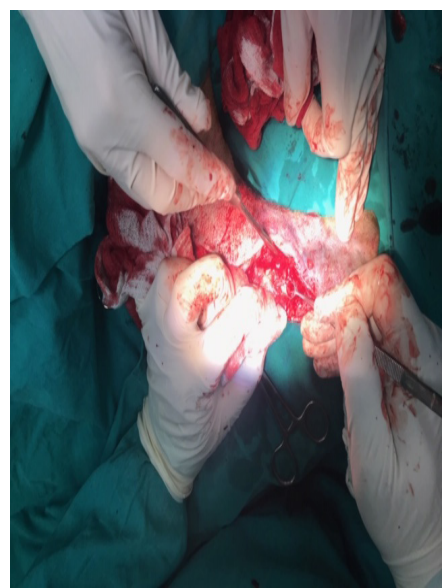

C

Figure 2. Intraoperative images of fracture with osteomyelitis after plate removal: femur (a and b), tibia (c) 
ance rates were documented for penicillin (55\%), and clindamycin (45\%).

There is no standard protocol for systemic and local antibiotic therapy that can be applied to every case, taking into account that antibiotic should always be tailored to antibiotic susceptibility of isolated bacteria for appropriate treatment. For the 329 cases of dogs with fractures treated surgically by osteosynthesis, antibiotic prophylaxis was performed with amoxicillin and potassium clavulanate $(223 / 68 \%)$, with ceftriaxone $(59 / 18 \%)$ or with ampicillin-sulbactam $(47 / 14 \%)$. For achieving high local antimicrobials concentration, intraoperatively we injected into the fracture site a single dose of lincomycin (30 $\mathrm{mg} / \mathrm{kg}$ ).

After specifying the diagnosis of osteomyelitis in all 11 registered cases, was performed removal of the metal implants (pins, plate or FEL) followed by surgical revision of the fracture fixation. The surgical revision consisted of wide debridement, curretage of devitalized tissues, removal of foreign bodies and osseous sequestration, reduction of fracture or alignment of brocken ends of the bone with the application of a loocking-plate with 4-6 screws $(9 / 82 \%)$ in a bridging fashion or in a combined intramedullary pin and loocking-plate fixation (1/0.9\%) (Figure 3).

In one case with chronic osteomyelitis with extensive suppurative and osteonecrotic lesions of the radius and ulna, amputation of the limb was needed.

Antibiotic treatment after surgical revision of the fracture fixation consisted of injection of ampicillin-sulbactam for 7 days $(30 \mathrm{mg} / \mathrm{kg}$ IV TID) and continued for 4-6 weeks by oral administration (Unasyn, $50 \mathrm{mg} / \mathrm{kg}$ BID) in 5 cases (46\%), ceftriaxone (25 mg/kg IV BID) for 7 days and continued for 2-3 weeks once daily with IM administration in 3 cases (27\%), and injectable administration of ampicillin-sulbactam $(30 \mathrm{mg} /$ kg IV TID) + metronidazole (20 mg/kg IV BID) for 7 days and continued for 4-6 weeks by oral administration (Unasyn, $50 \mathrm{mg} / \mathrm{kg}$ BID) in 2 cases $(18 \%)$.

All 10 cases undergoing surgical revision were cured 10-17 weeks after the revision surgery and 12-44 weeks after the initial orthopedic treatment.

We report an incidence rate of $3.34 \%$ of cases with osteomyelitis secondary to fractures repairs, to our knowledge there are no published veterinary clinical studies showing the frequency

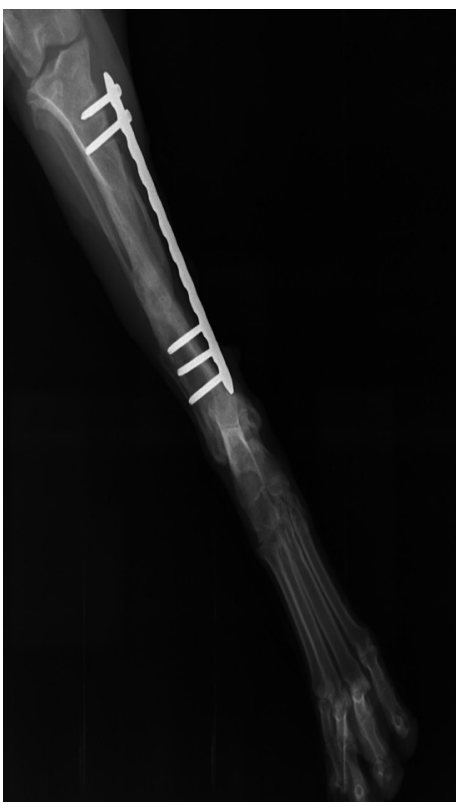

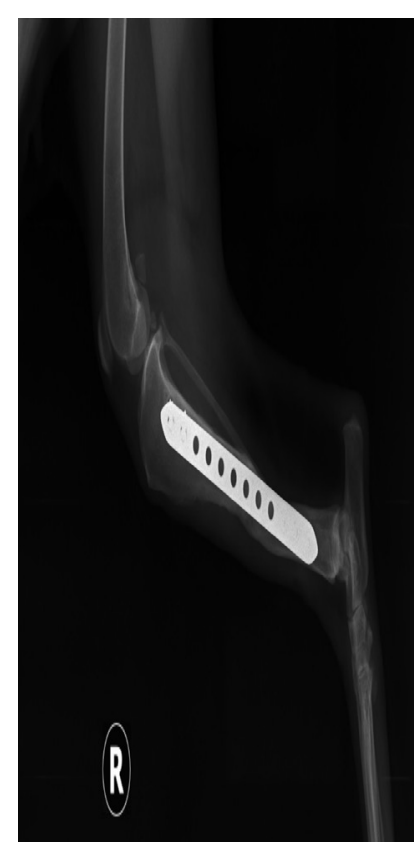

a

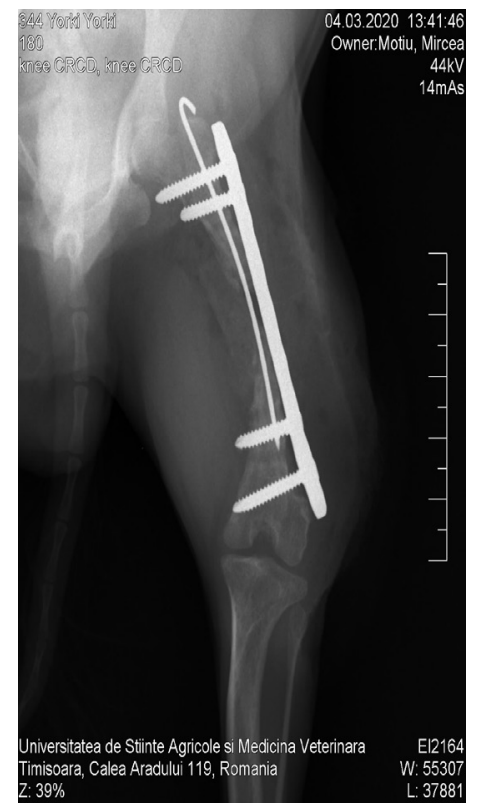

b

Figure 3. Surgical revision of the fixation of fracture-osteomyelitis: a loocking-plate with 5 screws in a bridging fashion, tibia - ventrodorsal and mediolateral views, radiographs made at 17 weeks after surgery

(a); intramedullary pin and loocking-plate fixation, femur - ventrodorsal view, radiographs made at 4 weeks after surgery (b) 
of osteomyelitis after fracture treatment. In human medicine in the United States of America, the infection rate is $5-15 \%$ in fracture fixation devices (Metsemakers et al. 2018).

In veterinary literature the most frequently reported fractured bones were the tibia $(43.33 \%$ - 69.23\% of long bone fractures), followed by the femur $(26.67 \%)$, the radius and ulna $(18.33 \%-63.63 \%)$ and the humerus $(11.67 \%)$ (Soontonvipart et al., 2003, Dvorak et al., 2000). In our study we found some variance of fracture location, femur being involved less often, only in $18 \%$, and the humerus more frequently, in a $18 \%$, but the frequency of tibial fractures is in agreement with those found in the literature (46\%). This appears due to less dense muscle covering the bone at these sites, it was found that infection always occurred in the tibia and the radius (Braden, 1991, Carek et al., 2001, Caywood, 1983, Dvorak et al., 2000, Johnson, 1994, Soontonvipart et al., 2003).

In this study the majority of injuries (64\%) were caused by a motor vehicle accident and a lower percentage by dog bites $(18 \%)$, that's in according to a study published by Siqueira et al. (2014), which reported in 78\% of the cases injuries caused by a motor vehicle accident, 17\% by dog bites and $5 \%$ by ascending infection due to pododermatitis.

All 11 of our cases presented exogenous osteomyelitis, the result of postoperative infection associated with open fractures (64\%), or closed fractures (36\%). Similar data were reported b y Siqueira et al. (2014), but after Kaim et al. (2001), incidence of osteomyelitis following open fractures are $2-16 \%$ depending significantly on the grade of trauma and the type of treatment administered.

In general, a single microorganism was more frequent than combined infections in exogenous forms of osteomyelitis, with predominance of Gram-positive bacteria (Jackson and Pacchiana, 2004). In the present study, isolation of only one microorganism was observed in 9 positive cultures $(82 \%)$, whereas $2(18 \%)$ cultures had more than one type of bacterium. The microorganisms most frequently isolated in this study were Staphylococcus spp. and Streptococcus spp. followed by Escherichia coli, and anaerobic bacteria. Similar studies have also reported Staphylococcus spp as the most common causal agent of osteomyelitis in companion animals, although other Gram-positive organisms such as Streptococcus and Gram negative bacteria including Escherichia coli, Pseudomonas spp., Proteus spp., Pasteurella multocida, and Klebsiella spp. were isolated (Fossum and Hulse, 1992; Jackson and Pacchiana, 2004; Johnson et al., 1984; Johnson, 1994; Muir and Johnson, 1992; Piermattei et al., 2006; Simionato et al., 2003). Soontonvipart et al. (2003) reported a high percentage of the bacterial isolates of Pseudomonas aeruginosa, stems resistant to cephalosporins.

The most effective drugs against isolates were amoxicillin-clavulanate potassium (79\%) and ceftriaxone (71\%). Siqueira et al. (2014), and Sen and Kilic (2012) report simillary dates. A study in dogs with osteomyelitis due to exposed bone, with or without fractures, also detected that Grampositive cocci were more sensitive to amoxicillinclavulanate potassium (Simionato et al., 2003).

In the 329 cases of dogs with fractures treated surgically by osteosynthesis, we used the antibiotic prophylaxis with amoxicillin and potassium clavulanate $(68 \%)$, ceftriaxone $(18 \%)$ or ampicillin-sulbactam (14\%) and intraoperatively, as a local antibiotic prophylaxis, the injection into the fracture site of a single dose of lincomycin. An alternative for local application of antibiotic is the use of carriers. Zalavras et al. (2004) use antibiotic-impregnated beads whenever dead space is present and reoperation is needed (second-look debridement, soft tissue coverage, bone grafting). Specifically, if a bone and soft tissue defect is present after debridement, as in severe open fractures and osteomyelitis, they placed the beads in the gap area.

The results of this study indicates that the protocol of simultaneous debridement, reduction and fixation of fractures, with the application of a locking plate in a bridging fashion or in a combined intramedullary pine with the fixing of the locking plate is a satisfactory method. In human medicine for the treatment of complicated mandibular fractures with osteomyelitis, Koury et al. (1994) reported similar data.

The high incidence in this study of osteomyelitis after fractures fixed with plates, rods or FEL (3.34\%) does not show a predisposition for bone infection of a certain type of fracture fixation. However, it is known that due to compression force applied between bone fragments to achieve the primary bone healing, this force may create the necrosis of bone and a higher risk of infec- 
tion. So it can be explained how fixation with plate and screw may contribute to high risk of infection (Cordero et al., 1994). Additionally, the compression dynamic plate (DCP) will increase the stress at the fracture site and it may produce bone necrosis. Because of lack of blood vessel supply at the fracture site, the cryptic infection may easily become manifest (Arens et al., 1996). The use in the current study, in the case of surgical revisions of cases of fractures with osteomyelitis secondary to osteosynthesis, of the blocking plates in a noncontact assembly shows that the above considerations can be largely removed, a fact also reported by studies in human medical journals (Eijer et al., 2001, Schlegel and Perren, 2006, Yildirim et al., 2017).

Similar data with our records on the incidence of osteomyelitis in fractures treated with FEL $(13.5 \%)$ are also reported by Johnson and Schaeffer (2008) and in the fracture treated with intramedullary pins in the reports of Muir and Johnson (1996), and Kaya et al. (2011).

This study shows high rates of healing of implant related infection in long bones in dogs (out of 11 cases, 10 cured) which were possible because new advances of veterinary orthopedics surgery linked with metalic implant devices manufacture, together with careful debridement that reduces bacterial load and clean the bacterial biofilm, microbiological cultures, in vitro antimicrobial susceptibility testing and systemic antibiotics administrations were effectively combined into the treatment strategy.

\section{Conclusion}

The results of this study indicate that the protocol of simultaneous debridement with implant removal, reduction, and rigid internal fixation of fracture is a satisfactory method for revision surgery in treatment of long bones fractures complicated with osteomyelitis.

Prospective studies and follow-up of larger numbers of patients would be necessary.

Acknowledgments. This research did not receive any specific grant from funding agencies in the public, commercial, or not-for-profit sectors.

\section{References}

1. Anderson A (2016). Osteomyelitis. In T. J. Gemmill, \& D. N. Clements (Eds.), BSAVA Manual of Canine and Feline Fracture Repair and Management (pp. 374-385). Quedgeley: BSAVA Library.

2. Arens ST, Hansis M, Schlegel U, Eijer, H, Printzen G, Ziegler WJ, Perren SM (1996). Infection after open reduction and internal fixation with dynamic compression platesClinical and experiment data. Injury, 27 (Suppl 3): 27-33.

3. Braden TD (1991). Postraumatic osteomyelitis. Vet Clin North Am: Small Anim Pract, 35: 781-811.

4. Bubenik LJ (2005). Infections of the skeletal system. Vet Clin North Am: Small Anim Pract, 35: 1093-1109.

5. Budesberg SC (2012). Osteomyelitis. In K. M. Tobias, \& S. A. Johnston (Eds.), Veterinary surgery small animal (pp. 669-675). St. Louis: Elsevier Saunders.

6. Buttin P, Bismuth C, Genevois JP, Carozzo C (2013). Contribution of computed tomography in chronic septic arthritis and osteomyelitis management in a dog. Revue Med Vet, 164: 226-229.

7. Callum WH (2006). Osteomielytis. In S. J. Birchard, \& R. G. Sherding (Eds.). Saunders Manual of Small Animal Practice-E-Book. Elsevier Health Sciences, https://doi. org/10.1016/B0-72-160422-6/50123-6

8. Carek PJ, Dickerson LM, Pharm D, Sack JL (2001). Diagnosis and Management of Osteomyelitis. Am Fam Physician, 63: 2413-2420

9. Caywood DD (1983). Osteomyelitis. Symp Orthop Dis, 13: 43-53.

10. Cordero J, Munuera L, Folgueira MD (1994). Influence of metal implants on infection. J Bone Joint Surg, 26: 1-56.

11. Dvorak M, Nekas A, Zatloukal J (2000). Complications of long bone fracture healing in dogs: functional and radiological criteria for their assessment. Acta Vet Brno, 69:107-114.

12. Eijer H, Hauke C, Arens S, Printzen G, Schlegel U, Perren SM (2001). PC-fix and local infection resistance-Influence of implant design on postoperative infection development, clinical and experimental results. Injury, 32 (Suppl 2): B38-43.

13. Emmerson T, Pead MJ (1999). Pathological fracture of the femur secondary to haematogenous osteomyelitis in a weimaraner. J Small Anim Pract, 40(5): 233-235.

14. Fossum TW, Hulse DA (1992). Osteomyelitis. Semin Vet Med Surg (Small Anim), 7: 85-97.

15. Jackson LC, Pacchiana PD (2004). Common complications of fracture repair. Clin Tech Small Anim Pract, 19: 168179.

16. Johnson AL, Schaeffer DJ (2008). Evolution of the treatment of canine radial and tibial fractures with external fixators. Vet Comp Orthopaed, 21(03): 256-261.

17. Johnson KA, Lomas GR, Wood AKW (1984). Osteomyelitis in dogs and cats caused by anaerobic bacteria. Aust Vet J, 61: 57-61.

18. Johnson KA (1994). Osteomyelitis in dogs and cats. J Am Vet Med Assoc, 205: 1882-1887. 
19. Kaim AH, Gross T, von Schulthess GK (2002). Imaging of chronic posttraumatic osteomyelitis. European Radiology, 12(5): 1193-1202.

20. Kaya M, Okumus Z, Yanmaz LE, Dogan E, Kirecci E (2011). Post-Traumatic Osteomyelitis and its Treatment in a Dog. Pak Vet J, 31: 371-374.

21. Koury ME, Perrott DH, Kaban LB (1994). The use of rigid internal fixation in mandibular fractures complicated by osteomyelitis. J Oral Maxil Surg, 52(11): 1114-1119.

22. Lio P, Paoletti N, Moni MA, Atwell K, Merelli E, Viceconti M (2012). Modelling osteomyelitis. BMC Bioinformatics, 13(Suppl 14):S12.

23. May C (2002). Management of bacterial osteomyelitis in dogs and cats. In Practice, 24(6): 330-337.

24. Metsemakers WJ, Kuehl R, Moriarty TF, Richards RG, Verhofstad MH, Borens O, Kates S, Morgenstern M (2018). Infection after fracture fixation: Current surgical and microbiological concepts. Injury, 49(3): 511-522.

25. Muir P, Johnson KA (1992). Anaerobic bacteria isolated from osteomyelitis in dogs and cats. Vet Surg, 21: 463466.

26. Muir P, Johnson KA (1996). Interlocking intramedullary nail stabilization of a femoral fracture in a dog with osteomyelitis. J Am Vet Med, 209(7): 1262-1264.

27. Piermattei DL, Flo G, Decamp C (2006). Treatment of acute and chronic bone infections. In W. O. Brinker, D. L. Piermattei, \& G. L. Flo (Eds), Handbook of small animal orthopedics and fracture repair (pp 177-184). Philadelphi: Saunders.

28. Quinn PJ, Markey BK, Leonard FC, Fitzpatrick ES, Fanning S, Hartigan PJ (2011). Veterinary microbiology and microbial disease. (2nd ed.). Chichester: Wiley-Blackwell, (Section VII).

29. Rabillard M, Souchu L, Niebauer GW, Gauthier O (2011). Haematogenous osteomyelitis: clinical presentation and outcome in three dogs. Vet Comp, 24(02): 146-150.
30. Schlegel U, Perren SM (2006). Surgical aspects of infection involving osteosynthesis implants: Implant design and resistance to local infection. Injury, 37 (Suppl 2): S67-73.

31. Schulz K (2007). Osteomyelitis. In T. W. Fossum (Ed.), Small animal surgery (pp. 1351-1356). St. Louis: Mosby.

32. Sen ZB, Kilic N (2012). Posttraumatic bacterial infections in extremities before and after osteosynthesis in dogs. Acta Sci Vet, 40(2): 1-7.

33. Simionato AC, Ramos MCC, Coutinho SDA (2003). Aerobic bacterial isolates and susceptibility to antimicrobial agents in canine osteomyelitis. Arq Bras Med Vet Zootec, 55: 148-154.

34. Siqueira EG, Rahal SC, Ribeiro MG, Paes AC, Listoni FP, Vassalo FG (2014). Exogenous bacterial osteomyelitis in 52 dogs: a retrospective study of etiology and in vitro antimicrobial susceptibility profile (2000-2013). Vet Quart, 34(4): 201-204.

35. Soontornvipart K, Necas A, Dvorak M (2003). Effects of metallic implant on the risk of bacterial osteomyelitis in small animals. Acta Vet Brno, 72: 235-247.

36. Stein A, Bataille JJ, Drancourt M, Curvale G, Argenson JN, Groulier P, Raoul TD (1998). Ambulatory treatment of multidrug-resistant Staphylococcus infected orthopaedic implants with high-dose oral co-trimazole (Trimethoprim-Sulfamethoxazole). Antimicrob Agents Ch, 42: 3086-3091.

37. Woodard JC, Riser WH (1991). Morphology of fracture nonunion and osteomyelitis. Vet Clin North Am: Small Anim Pract, 21: 813-844.

38. Yildirim A, Kapukaya A, Mertsoy Y, Yigit S, Cacan MA, Atic R (2017). Management of open fractures using a noncontact locking plate as an internal fixator. Indian J Orthop, 51(3): 312.

39. Zalavras CG, Patzakis MJ, Holtom P (2004). Local antibiotic therapy in the treatment of open fractures and osteomyelitis. Clin Orthop Relat R, 427: 86-93. 